\title{
An Efficient Multicast Routing Protocol in Multi-rate Wireless Ad Hoc Networks ${ }^{\star}$
}

\author{
Kyung-Jin Bae ${ }^{1}$, Dong-Hee Kwon ${ }^{2}$, Woo-Jae Kim² ${ }^{2}$, and Young-Joo Suh ${ }^{2}$ \\ ${ }^{1}$ S/W Laboratory, Mobile \& Communication Division \\ Information \& Communications Business, Samsung Electronics \\ Suwon, Korea \\ kyungjin80. bae@samsung.com \\ ${ }^{2}$ Department of Computer Science and Engineering \\ Pohang University of Science and Technology (POSTECH) \\ Pohang, Korea \\ $\{$ ddal, hades15, yjsuh\}@postech.ac.kr
}

\begin{abstract}
In wireless ad hoc networks, many multicast routing protocols are proposed such as ODMRP, MAODV, etc. In general, the IEEE 802.11 standard is assumed as a MAC protocol in those protocol and multicast data packets are transmitted at the basic transmission rate which is $2 \mathrm{Mbps}$ at the IEEE 802.11 or $802.11 \mathrm{~b}$. However, if the multicast packet can be transmitted at higher transmission rates than $2 \mathrm{Mbps}$ in a multi-rate WLAN such as IEEE 802.11b, we can achieve higher throughput gain and lower transmission delay than those at $2 \mathrm{Mbps}$ transmission rate. In this paper, we propose a new multicast routing protocol in multirate wireless ad hoc networks. In the proposed protocol, we design a multicast mesh creation method considering multiple transmission rates between any two nodes in the ad hoc networks. We also design a multicast mesh maintenance scheme when the transmission rate between two nodes comprising a multicast mesh is changed because of node mobility. The performance of the proposed protocol is compared with ODMRP using ns2 simulation, and the proposed protocol shows higher packet delivery ratio and lower end-to-end delay than the ODMRP.
\end{abstract}

\section{Introduction}

Wireless ad hoc networks are self-organizing wireless networks composed of mobile nodes with no fixed infrastructure or central administration. Because of the limited radio propagation range of wireless devices, each node must have the function that forwards data packets to other nodes. Therefore, wireless ad hoc networks have more constraints than infrastructure networks such as scarce resources, a dynamic topology, and lack of any established backbone infrastructure. In these networks, a challenging issue has been the development of multicast

\footnotetext{
* This research was supported by the MIC(Ministry of Information and Communication), Korea, under the ITRC(Information Technology Research Center) support program supervised by the IITA(Institute of Information Technology Assessment) (IITA-2005-C1090-0501-0018).
} 
routing protocols that suit the features of wireless ad hoc networks. Multicast is an efficient transmission method to deliver data packets to multiple destinations. There have been many multicast routing protocols in wired networks. But they are not well-suited for wireless ad hoc networks. In wireless ad hoc networks, the constructed multicast tree can be fragile and should be reconstructed which causes an excessive overhead to the network. Because of this highly-dynamic topology of wireless ad hoc networks, several multicast routing protocols have been proposed $[8,10,12,14]$.

Among them, On Demand Multicast Routing Protocol (ODMRP) [14] establishes and maintains a multicasts mesh structure as demand basis by the source to deal with mobility of nodes. It is similar to on-demand unicast routing protocols because there are the query phase and reply phase to find routes. While a multicast source has packets to send, it periodically broadcasts a JOIN_QUERY message to the entire network to find multicast members. This periodic transmission sets up a multicast forwarding state when the source first starts sending multicast data, and recovers from partitions and breaks in the forwarding tree as a result of node movement. When a node receives a non-duplicate JOIN_QUERY message, it stores the upstream node address in its route table and rebroadcasts the JOIN_QUERY message. When a multicast member receives a JOIN_QUERY message, it creates or updates a source entry in its member table. While valid entries exist in the member table, JOIN_TABLE is broadcast periodically to the neighbors. When a node receives a JOIN_TABLE, it checks if it is a part of a forwarding group or not comparing its own address and next hop addresses of the JOIN_TABLE. If it is a member of a forwarding group, the node sets the FG_FLAG and broadcasts its own JOIN_TABLE. This process constructs or updates the mesh structure of forwarding groups. After the construction of multicast mesh structure, multicast data are transmitted from the source to multicast members by rebroadcasting of forwarding nodes.

In general, the IEEE 802.11 standard is assumed as a MAC protocol in wireless ad hoc networks. The IEEE 802.11 [2] is the standard for wireless LANs supporting the data transmission rates of 1 and $2 \mathrm{Mbps}$. There are some PHY extensions to provide higher bandwidth to users. The IEEE 802.11b [1] is a high-rate PHY extension supporting 5.5 and $11 \mathrm{Mbps}$ data transmission rate in 2.4GHz band. The IEEE $802.11 \mathrm{a}$ [3] is a new PHY extension that operates at the $5 \mathrm{GHz}$ band with Orthogonal Frequency Division Multiplexing (OFDM) radio and provides eight transmission rates from $6 \mathrm{Mbps}$ to $54 \mathrm{Mbps}$. Another PHY extension, the IEEE 802.11g standard [5], which operates in the same frequency band as IEEE 802.11b is designed for compatibleness with the IEEE 802.11b devices while providing the data rates up to $54 \mathrm{Mbps}$ with OFDM radio.

Although the IEEE 802.11 protocol family can support multiple transmission rates as addressed previously, only single base transmission rate is used in a multicast data transmission. This is because there are many neighbors having different data transmission rates and there is no algorithm to know the rate information of neighbors to which it forwards multicast data. Also, multicast data delivery is not guaranteed by the RTS/CTS exchange and, therefore, the 
multicast packet should be transmitted at a high reliable channel. However, if the multicast packet can be transmitted at higher transmission rates than base transmission rate in a multi-rate WLAN, we can achieve higher throughput gain and lower transmission delay than those at the base transmission rate.

In this paper, we propose a new multicast routing protocol in multi-rate wireless ad hoc networks. In the proposed protocol, we design a multicast mesh creation method considering multiple transmission rates between any two nodes in the ad hoc networks. We also design a multicast mesh maintenance scheme when the transmission rate between two nodes comprising a multicast mesh is changed because of node mobility. The performance of the proposed protocol is compared with ODMRP using ns2 simulation, and the proposed protocol shows higher packet delivery ratio and lower end-to-end delay than the ODMRP.

\section{Backgrounds}

The rate adaptation technique [6] is widely used in wireless networks to adapt modulation and coding schemes according to a channel condition. Generally, higher data rates are achieved by more efficient modulation techniques. However, highly efficient modulation techniques need denser modulation encodings which may result in high bit error rate. Since a modulation technique determines the ability to preserve the high accuracy of the encoded data, it is important to select the proper modulation scheme according to the current channel condition. In practice, there are many factors to affect on the channel condition, and the wireless channel conditions may vary significantly. Signal-to-Noise Ratio (SNR) is widely used as the factor of the channel condition. According to SNR, thus, the suitable modulation technique can be selected to achieve a balance between data transmission rate and the bit error rate.

Among several multi-rate extensions of IEEE 802.11, we discuss a multi-rate extension of IEEE 802.11b since it is widely used. In the IEEE 802.11b standard [2], a modulation technique called direct sequence spread spectrum (DSSS) is used to support multi-rate schemes. It is specified in the $2.4 \mathrm{GHz}$ band and provides additional 5.5 and $11 \mathrm{Mbps}$ data rates. Therefore, a node can decide the transmission data rate of $1 \mathrm{Mbps}, 2 \mathrm{Mbps}, 5.5 \mathrm{Mbps}$, and $11 \mathrm{Mbps}$ according to the SNR. All control frames (RTS, CTS, ACK and Management frames) in the IEEE 802.11b specification are transmitted at the base rate to correctly provide the virtual carrier sense mechanism. All broadcast and multicast frames are also transmitted at the base rate since those frames cannot be protected by the RTS-CTS exchange mechanism.

\section{Motivation}

A routing protocol usually establishes routes based on minimal hop count. In other words, it establishes the shortest path as a route. But it can be a nonoptimal route in the environment which supports multi-rate scheme. In multirate WLANs, the data transmission time at a higher rate is generally much 


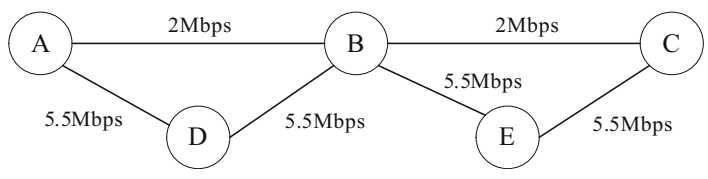

Fig. 1. Multi-rate ad hoc networks

shorter than the transmission time at a lower rate $[9,11]$. Figure 1 shows an example. There are 5 nodes, the source is $\mathrm{A}$ and the destination is C. Each link has different data rates of $2 \mathrm{Mbps}$ and $5.5 \mathrm{Mbps}$. In this situation, existing routing protocols will establish a route $\mathrm{A} \rightarrow \mathrm{B} \rightarrow \mathrm{C}$ because it is the shortest path. But it has a base data rate of 2 Mbps. If we select a route through $A \rightarrow D \rightarrow B \rightarrow E \rightarrow C$, we can use higher link bandwidth by using a higher data rate than $2 \mathrm{Mbps}$. This may increase the number of hop count and result in increase of channel access overhead. But it can reduce the data transmission time and we can achieve better throughput.

When this multi-rate scheme is applied to multicast, there are many neighbors having different data transmission rates and there is no algorithm to know neighbors' rate information. Hence we propose a technique which makes a forwarding node knows the sending data rates as well as a route selection algorithm to find a better route based on the data rate.

\section{Proposed Protocol}

\subsection{Creation of a Multicast Mesh Structure and Packet Forwarding}

In the proposed protocol, group membership and multicast routes are established and updated by the source on-demand. Just like ODMRP, the proposed protocol is based on a multicast mesh structure to deal with mobility of mobile nodes. A mesh structure is robust to mobility of nodes since it has multiple routes from the source to receivers.

The proposed protocol has a query and a reply phase. While a multicast source has packets to send, it periodically broadcasts a member advertising packet (JOIN_QUERY) to the entire network. This JOIN_QUERY packet is transmitted at a base transmission rate $(2 \mathrm{Mbps})$ for guaranteeing every node in the network to receive it, since the JOIN_QUERY packet plays a role that informs the rate information to other nodes receiving it. When a node receives a non-duplicate JOIN_QUERY packet, it stores the upstream node ID and determines the transmission rate of a previous hop with the help of a MAC protocol. After that, the node attaches the determined data transmission rate on the JOIN_QUERY packet and rebroadcasts it. When a multicast member receives the JOIN_QUERY packet, it selects the best route based on rate information in the JOIN_QUERY packet. In other words, a multicast receiver selects the route which has the smallest number of $2 \mathrm{Mbps}$ links based on the rate information in the JOIN_QUERY packet. If the number of $2 \mathrm{Mbps}$ links in the routes is the 
same, the best route is determined by the number of $5.5 \mathrm{Mbps}$ links and if the number of $5.5 \mathrm{Mbps}$ links is also the same, the best route is determined by the number of $11 \mathrm{Mbps}$ links. If the number of $11 \mathrm{Mbps}$ links is the same, a multicast receiver selects the route with minimal hop count. Through this simple method, multicast receivers can choose the route which has a higher data rate.

After the multicast receiver selects the route, it creates or updates the source entry in its member table. If the multicast receiver has valid entries in its member table, JOIN_REPLY packets containing the member table information are broadcast periodically to the neighbors. When a node receives a JOIN_REPLY packet, it checks if one of next node IDs in a JOIN_REPLY packet matches with its own ID. When two IDs are same, the node realizes that it is on the path from the source to the receiver and thus the node is part of a forwarding group. Therefore, the node sets itself as the forwarding node, stores the data rate of the next hop which multicast data will be forwarded, and broadcasts its own JOIN_REPLY packet. The JOIN_REPLY packet is, thus, propagated by each forwarding group member until it reaches the multicast source. This process constructs or updates the routes from the source to receivers and builds a mesh structure of the forwarding group.

Figure 2 shows the process of building a mesh structure, where $\mathrm{S}$ is the multicast source and R1, R2, and R3 are multicast receivers. Each link between two nodes has a data rate among $2 \mathrm{Mbps}, 5.5 \mathrm{Mbps}$, and $11 \mathrm{Mbps}$. When $\mathrm{S}$ is starting to send multicast data, it sends JOIN_QUERY packets periodically to find a route. As shown in Figure 2, the data transmission rate of previous hop is attached on each JOIN_QUERY packet. When multicast receivers receive JOIN_QUERY packets, they select the best route and send periodically their JOIN_REPLY packets to neighbors. In Figure 2, R1 selects a $<5.5,5.5>$ JOIN_QUERY packet and R2 and R3 select $<11,11,11,5.5>$ and $<11,11>$ JOIN_QUERY packets, respectively.

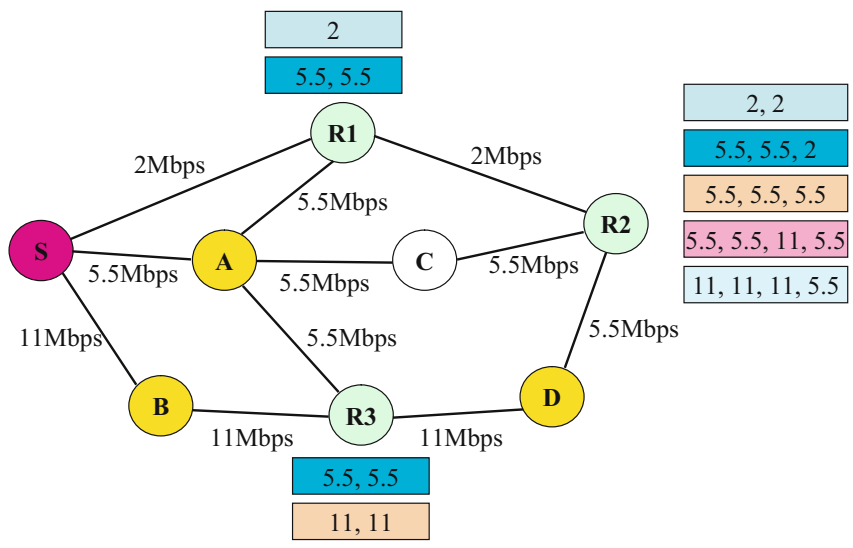

Fig. 2. Creation of a multicast mesh structure 
Once a multicast mesh structure is created, the multicast source starts to send multicast data. But the source node selects a data transmission rate carefully when it sends multicast data, because there can be two or more neighbors which have different data transmission rates for receiving multicast data. If the data transmission rates to neighbors are different with each other, it happens that some neighbors cannot receive multicast data successfully due to different transmission rates. Thus, the sending data transmission rate is selected as the lowest data transmission rate among those of neighbors which have to receive multicast data. When the node receives multicast data, it checks if it is a forwarding node or not. If the receiving node is a forwarding node, it chooses a sending data transmission rate and forwards multicast data at the selected data transmission rate.

\subsection{Maintenance of a Multicast Mesh Structure}

The data transmission rate of a link can be changed due to the mobility of mobile nodes. The change of data transmission rate prevents a forwarding node from receiving multicast data successfully and results in a failure of packet delivery. Thus, the change of data transmission rate should be reported immediately when it is detected. When a node recognizes the change of data transmission rates on a previous link, it sends a warning message containing changed data transmission rate information to previous node in a base transmission rate. The node receiving the warning message compares its sending data transmission rate and data transmission rate information in the warning message.

The intermediate node receiving the warning message updates its sending rate $\left(R_{O}\right)$ to the value $\left(R_{N}\right)$ in the warning message as follows:

$-R_{O}<R_{N}$ : If there are no neighbors that have a lower one, the node updates its sending data transmission rate to a value in the warning message. If there are neighbors that have lower data transmission rate than the $R_{N}$, the node just discards the warning message.

$-R_{O}>R_{N}$ : The node updates its sending data transmission rate to a value in the warning message.

$-R_{O}=R_{N}$ : The node discards a warning message.

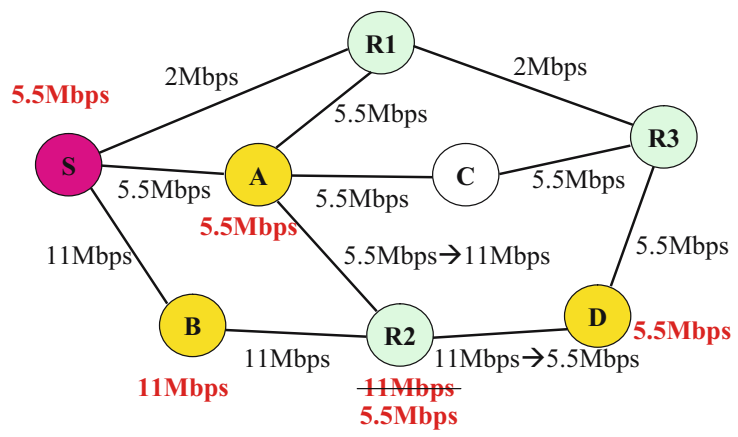

Fig. 3. Maintenance of a multicast mesh structure 
Figure 3 shows an example of changing the data transmission rate. In Figure 3, the link between $\mathrm{A}$ and $\mathrm{R} 2$ is changed from 5.5Mbps to $11 \mathrm{Mbps}$. In this case, R2 sends a warning message to A and A compares its sending data rate with $11 \mathrm{Mbps}$ included in the warning message, and A discards the warning message since A has a neighbor with the data rate of 5.5Mbps (R1). If the link between $\mathrm{R} 2$ and $\mathrm{D}$ is changed from $11 \mathrm{Mbps}$ to $5.5 \mathrm{Mbps}$, node $\mathrm{D}$ sends warning messages to $\mathrm{R} 2$, and $\mathrm{R} 2$ should update its sending rate from $11 \mathrm{Mbps}$ to $5.5 \mathrm{Mbps}$.

\section{Performance Evaluation}

\subsection{Simulation Environment}

We perform simulations using the NS2 network simulator [13]. The radio model is based on the IEEE 802.11 supporting multi-rate scheme, which provides a various data transmission rates of $2 \mathrm{Mbps}, 5.5 \mathrm{Mbps}$, and $11 \mathrm{Mbps}$ and a nominal transmission range of $250 \mathrm{~m}$. We randomly placed 50 mobile nodes in a $1500 \mathrm{~m}$ $\times 300 \mathrm{~m}$ network area and let them move freely at a given maximum speed of $20 \mathrm{~m} / \mathrm{s}$ during simulation time of 300 seconds. The nodes in our simulations move according to the Random Waypoint model [15]. For each maximum node movement speed, we run a simulation with 5 different pause times of $0,30,60$, 120, and 300 seconds. A pause time of 0 represents a network in which all nodes move continuously, while a pause time of 300 represents a stationary network. For each of these pause times and maximum node movement speeds, we randomly generate 5 different scenarios, and we present here the average over those 5 scenarios. We generate a constant bit rate (CBR) traffic in the application layer with 5 and 20 packets per second packet generation rates of 128 and 1024 bytes packet size. There is one source, one multicast group, and 15 multicast receivers. The multicast source starts sending data and multicast receivers join a group at uniformly randomly generated times between 0 and 60 seconds.

We evaluate the performance of the proposed protocol and compare it to that of the ODMRP using the following metrics; Packet delivery ratio - average number of data packets actually received by multicast receivers over the number of data packets originated by multicast source. End-to-end delay - average time elapsed between when a multicast data packet is originated by a source and when it is successfully received by a multicast receiver. Normalized control overhead total number of data and control packets transmitted by any node in the network, divided by the total number of data packets received by all multicast receivers.

\subsection{Simulation Results}

The packet delivery ratio as a function of the pause time is shown in Figure 4 . As shown in the figure, we can see that the delivery ratio is decreased as the pause time is decreases. When the pause time is small, node mobility may be increased. Therefore, the route between source and receiver can be easily broken. This means that more packets are dropped during the route reconstruction when 


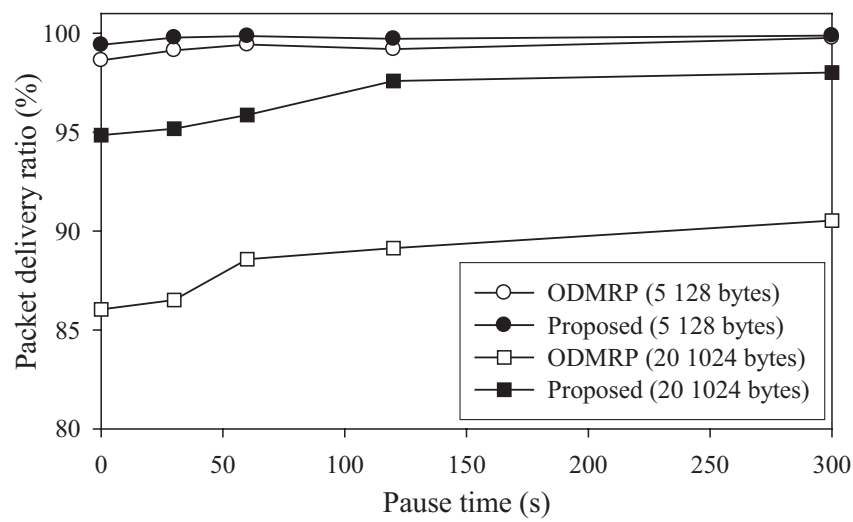

Fig. 4. Packet delivery ratio

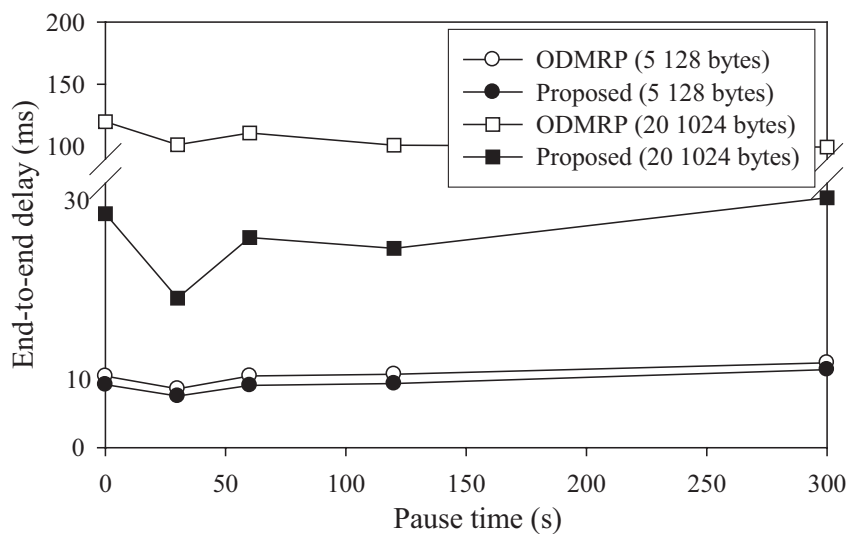

Fig. 5. End-to-end delay

the pause time is small. As shown in the figures, the proposed protocol shows better performance in packet delivery ratio than the ODMRP. This is because there is less number of dropped packets in the proposed protocol by using higher transmission rates than the ODMRP. This phenomenon is more dominant in the case of heavy network load, i.e., the packet generation rate is 20 packets per second and the packet size is 1024 bytes.

Figure 5 shows the end-to-end delay as a function of the pause time. The proposed protocol shows lower end-to-end transmission delay than the ODMRP because the proposed protocol selects better routes which can use higher data transmission rates. The proposed protocol show much better performance than ODMRP when the traffic load is high (when the packet generation rate is 20 packets per second and the packet size is 1024 bytes). It is due to the fact that the proposed protocol experiences less queuing delay by using higher data transmission rate than ODMRP, and thus the proposed protocol spends less 


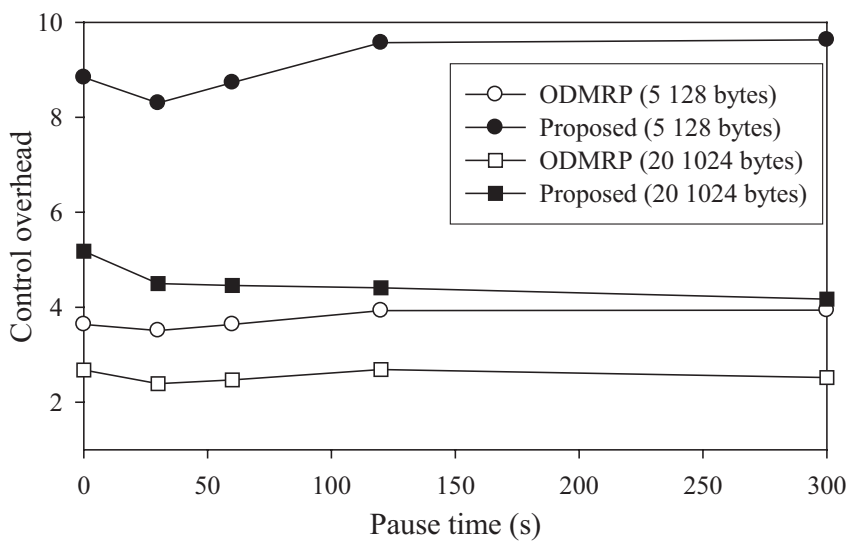

Fig. 6. Normalized control overhead

time to transmit multicast data packets. Therefore, the end-to-end delay of the proposed protocol is less than that of ODMRP.

Figure 6 shows the normalized control overhead as a function of the pause time. In the figure, we can see that the normalized control overhead is constant regardless of the pause time, because control packets such as JOIN_QUERY and JOIN_TABLE are transmitted periodically in both of the protocols. And the reason that the proposed protocol has more control overhead than the ODMRP is as follows. In the proposed protocol, JOIN_TABLEs are broadcast in response of every JOIN_QUERY because JOIN_QUERY that arrives later can have better route, i.e. route at higher data transmission rate. However, in the ODMRP, the multicast member only broadcasts JOIN_TABLEs in response to the JOIN_QUERY arriving at first. Also, in the proposed protocol, nodes send a warning message when the data transmission rate of a link is changed. Therefore the normalized control overhead of the proposed protocol is slightly higher than that of the ODMRP.

\section{Conclusion}

In wireless ad hoc networks, many multicast routing protocols are proposed such as ODMRP, MAODV, etc. In general, multicast data packets are transmitted at the basic transmission rate which is $2 \mathrm{Mbps}$ at the IEEE 802.11 or $802.11 \mathrm{~b}$. This is because a multicast packet delivery is not guaranteed by the RTS/CTS exchange and there can be many neighbors with different transmission rates. However, if the multicast packet can be transmitted at higher transmission rates than 2Mbps in a multi-rate WLAN such as IEEE 802.11b, we can achieve higher throughput gain and lower transmission delay than those at $2 \mathrm{Mbps}$ transmission rate. In this paper, we propose a new multicast routing protocol in multi-rate wireless ad hoc networks. In the proposed protocol, we design a multicast mesh creation method considering multiple transmission rates between any two nodes 
in the ad hoc networks. We also design a multicast mesh maintenance scheme when the transmission rate between two nodes comprising a multicast mesh is changed because of node mobility. According to our simulation results, the proposed protocol shows higher packet delivery ratio and lower end-to-end delay compared with ODMRP, while shows increased control overhead. The proposed protocol can be good for a real time traffic which needs a high packet delivery ratio and short end-to-end delay, especially in the network which has heavy load.

\section{References}

1. IEEE 802.11b, Part 11:Wireless LAN Medium Access Control (MAC) and Physical Layer (PHY) Specifications: High-Speed Physical Layer Extension in the $2.4 \mathrm{GHz}$ Band, supplement to IEEE 802.11 Standard, Sept. 1999.

2. IEEE 802.11, Wireless LAN Medium Access Control (MAC) and Physical Layer (PHY) Specifications, Standard, IEEE, Aug. 1999.

3. IEEE 802.11a, Part 11:Wireless LAN Medium Access Control (MAC) and Physical Layer (PHY) Specifications: High-Speed Physical Layer in the 5GHz Band, supplement to IEEE 802.11 Standard, Sept. 1999.

4. A. Kamerman and L. Monteban, "WaveLAN 11: A highperformance wireless LAN for unlicensed band," Bell Labs Technical Journal, 1997.

5. IEEE 802.11g, Part 11:Wireless LAN Medium Access Control (MAC) and Physical Layer (PHY) Specifications: Further Higher Data Rate Extension in the $2.4 \mathrm{GHz}$ Band, amendment to IEEE 802.11 Standard, Jun. 2003.

6. G. Holland, N. Vaidya and P. Bahl, "A rate-adaptive MAC prctocol for multi-hop wireless networks," ACM MOBICOM, 2001.

7. IEEE 802.11b, "Part 11:Wireless LAN Medium Access Control (MAC) and Physical Layer (PHY) Specifications: High-Speed Physical Layer Extension in the 2.4GHz Band," Standard, IEEE, 1999.

8. J. J. Garcia-Luna-Aceves and E. L. Madruga, "The core-assisted mesh protocol Garcia-Luna-Aceves," IEEE JSAC, 1999.

9. Yongho Seok, Jaewoo Park and Yanghee Choi, "Multi-rate Aware Routing Protocol for Mobile Ad Hoc Networks," IEEE VTC Spring, 2003.

10. E. Bommaiah, M. Liu, A. McAuley and R. Talpade, "AMRoute: Ad hoc multicast routing protocol," Internet draft, draft-talpade-manet-amroute-00.txt, IETF, 1998.

11. H. Zhu and G. Cao, "On improving the performance of IEEE 802.11 with multihop concepts," IEEE ICCCN, 2003.

12. C. W. Wu and Y. C. Tay, "ARMRIS: A multicast protocol for ad hoc wireless networks," IEEE MILCOM, 1999.

13. K. Fall and K. Varadhan, The ns Manual, available from http://www.isi.edu/ nsnam/ns, 2003

14. S. J. Lee, W. Su and M. Gerla, "On-demand multicast routing protocol (ODMRP) for ad hoc networks," Internet draft, draft-ietf-manet-odmrp-02.txt, IETF, 2000.

15. C. Bettstetter, H. Hartenstein, and X. Perez-Costa., "Stochastic properties of the random waypoint mobility model," ACM/Kluwer Wireless Networks, 2004. 